\title{
A New 3D Method for Reactor Core Vibration Based on Silicon Steel Lamination Rules and Application in UHV Shunt Reactors
}

\author{
Fangcheng Lü,, ${ }^{1,2}$ Jiayi Guo $\mathbb{D}^{1,2}$ Leilei Niu, ${ }^{1,2}$ Jianghai Geng, ${ }^{1}$ and Yirui Pan ${ }^{1}$ \\ ${ }^{1}$ Hebei Provincial Key Laboratory of Power Transmission Equipment Security Defense, North China Electric Power University, \\ Baoding, Hebei Province 071003, China \\ ${ }^{2}$ State Key Laboratory of Alternate Electrical Power System with Renewable Energy Sources, \\ North China Electric Power University, Beijing 102206, China \\ Correspondence should be addressed to Jiayi Guo; guojiayi@ncepu.edu.cn
}

Received 7 July 2019; Revised 19 September 2019; Accepted 5 October 2019; Published 23 October 2019

Academic Editor: Denis Benasciutti

Copyright ( 2019 Fangcheng Lü et al. This is an open access article distributed under the Creative Commons Attribution License, which permits unrestricted use, distribution, and reproduction in any medium, provided the original work is properly cited.

\begin{abstract}
A new three-dimensional (3D) analysis method is proposed as the existing two-dimensional (2D) method has low accuracy in analysing the vibration characteristics of oil-immersed shunt reactors, such as ultrahigh-voltage (UHV) shunt reactors. First of all, a set of 3D laminated coordinate systems was defined based on silicon steel lamination rules, in which the anisotropy of magnetic properties for laminated silicon steel in the rolling direction (RD), the transverse direction (TD), and the lamination direction (LD) were considered. Then, the mapping between laminated coordinate systems and space rectangular coordinate system was established to unify the parameters in different laminated coordinate systems. With the mapping, the anisotropy of the magnetic properties in the laminated coordinate systems was transformed into a rectangular coordinate system. Next, two sets of comparative studies between the new 3D method and the traditional 2D method were carried out, which show that the 3D method has high precision and a wide application range. Finally, the relationship between air gap number and core vibration of UHV shunt reactors was studied by the new 3D method. The results show that, as the number of air gaps increases, the magnetic flux density and the total force area of Maxwell force are increased, resulting in the intensification of core vibration. The conclusions of this paper are helpful for the design of large oil-immersed reactors.
\end{abstract}

\section{Introduction}

A shunt reactor, which compensates for long-distance line capacitance and suppressing the increase of power frequency, is one of the important items of equipment in AC transmission projects. Excessive vibration is one of the most common problems in the operation of shunt reactors due to the air gaps in the core limb that provide large inductance [1].

The vibration of the core (iron yoke and core limb) is one of the most important causes of shunt reactor vibration [1-9]. The elastic deformation of the core in the magnetic field due to Maxwell force and magnetostriction is considered to be the main reason for shunt reactor core vibration [2]. 2D methods have been widely used to study this $[3,8]$. In this type of method, the core limb is considered to have the same thickness in the $z$-direction. The difference in magnetic properties (magnetisation curves, magnetostrictive characteristic parameters, and so on) of the core material is called anisotropy. The 3D anisotropy (in the direction of $\mathrm{RD}$, $\mathrm{TD}$, and $\mathrm{LD}$ ) has been simplified into $2 \mathrm{D}$ (RD and TD) in the 2D method. The 2D method has high accuracy in the study of small dry reactors with cuboid core limbs [3, 4]. However, the core limb of many large oil-immersed shunt reactors such as UHV shunt reactors is a cylinder with laminated silicon steel, which is very different from the core limb of a small dry reactor. Thus, a new and wider range of method is needed.

The air gap in the core limb is one of the main reasons why the shunt reactor vibration is greater than in other similar items of equipment such as transformers. Adjusting the air gaps can be a useful method to reduce the vibration of shunt reactors. The method of adding air gaps in the yokes and adjusting the hardness of the air gap material was proposed by 
Gao et al. [4]. The method of filling the air gap by a novel method inserting negative giant magnetostrictive material (NGMM) was proposed by Yan et al. [5]. The aforementioned research has paid more attention to the relationship between air gap material and vibration. The influence of air gap structure on core vibration remains to be studied.

Here, a set of 3D laminated coordinate systems was constructed based on the lamination structure of the shunt reactor core, and a new $3 \mathrm{D}$ analysis method for the core vibration of shunt reactor column structure was proposed. Then, the 3D method was compared with the traditional 2D method by two sets of comparative studies; this method has a wider range of applications than the $2 \mathrm{D}$ method. Finally, the 3D method was applied to study the relationship between air gap number and core vibration of a UHV shunt reactor, which is typically a large oil-immersed reactor.

\section{Methods}

2.1. Laminated Coordinate Systems. The vibration of the core (iron yoke and core limb) is one of the most important causes of the shunt reactor vibration. The core of the shunt reactor is composed of laminated silicon steel sheets, whose magnetic properties are related to the direction of lamination [6]: there are different lamination directions in different parts of the core. According to the rules governing the lamination of silicon steel sheets, three mutually perpendicular axes including RD, TD, and LD can be set; on this basis, a set of $3 \mathrm{D}$ coordinate systems is created. The anisotropy of magnetic properties for laminated silicon steel can be expressed more clearly thereby.

There are two types of shunt reactor, dry type with small capacity in Figure 1(a) and oil-immersed type with large capacity in Figure 1(b) [3, 7]. Core limb and iron yoke are the two main parts of the reactor core. The core limb includes core discs and air gaps (marble or ceramics). The core discs for small dry reactors are usually cuboid, and core discs for large oil-immersed shunt reactors are generally cylindrical. The mapping between the laminated coordinate systems and the spatial rectangular coordinate system is as shown in Table 1.

The lamination rules of the cores at different positions are different, so the number of lamination coordinate systems is large, which entails onerous computations. It is necessary to define a global coordinate system (a space rectangular coordinate system here), which is used to unify parameters of different coordinate systems. Any vector has two different representations belonging to the two coordinate systems. The mapping between these two representations is beneficial to the calculation of the vibration parameters of the reactor.

2.1.1. Large Oil-Immersed Shunt Reactors. As shown in Table 1, for any vector $\mathbf{M}$ (in $3 \mathrm{D}$ coordinates) in the iron yokes of large oil-immersed shunt reactors, the mapping between the two coordinate systems satisfying equation (1) (top and bottom yokes) and equation (2) (side yokes) is as follows:

$$
\begin{aligned}
& \left\{\begin{array}{l}
M_{\mathrm{ld}}=M_{x}, \\
M_{\mathrm{td}}=M_{z}, \\
M_{\mathrm{rd}}=M_{y},
\end{array}\right. \\
& \left\{\begin{array}{l}
M_{\mathrm{ld}}=M_{x}, \\
M_{\mathrm{td}}=M_{y}, \\
M_{\mathrm{rd}}=M_{z} .
\end{array}\right.
\end{aligned}
$$

The core disc (Figure 2) of a large oil-immersed shunt reactor is generally formed by splicing a plurality of fanshaped laminated silicon steels. The fan-shaped laminated silicon steel is formed by stacking a plurality of rectangular silicon steel sheets with different lengths. The laminated coordinate system is related to the location of each sheet, and different laminated silicon steels belong to different $3 \mathrm{D}$ laminated coordinate systems. Taking the fanned laminated silicon steel marked with the border in Figure 2 as an example, the dotted lines indicate the LD (coinciding with the centreline of the sector), and the TD of the laminated coordinate system in which the laminated silicon steel is located. The solid lines indicate the $x$ and $y$-axes in a 3D space rectangular coordinate system. The $\mathrm{RD}$ is recombined with the $z$-axis perpendicular to the core disc plane. The angle between the $x$-axis and the LD is $\alpha$. The mapping of any vector $\mathbf{M}$ (in $3 \mathrm{D}$ coordinates) in the core discs between the two coordinate systems is as follows:

$$
\left\{\begin{array}{l}
M_{\mathrm{ld}}=M_{x} \cos \alpha+M_{y} \sin \alpha, \\
M_{\mathrm{td}}=-M_{x} \sin \alpha+M_{y} \cos \alpha, \\
M_{\mathrm{rd}}=M_{z} .
\end{array}\right.
$$

2.1.2. Small Dry Reactors. As shown in Table 1, the mapping for dry reactors can be performed by equations (4) (yokes) and (5) (core discs).

$$
\begin{aligned}
& \left\{\begin{array}{l}
M_{\mathrm{ld}}=M_{z}, \\
M_{\mathrm{td}}=M_{y}, \\
M_{\mathrm{rd}}=M_{x},
\end{array}\right. \\
& \left\{\begin{array}{l}
M_{\mathrm{ld}}=M_{z}, \\
M_{\mathrm{td}}=M_{x}, \\
M_{\mathrm{rd}}=M_{y},
\end{array}\right.
\end{aligned}
$$

where $M_{\mathrm{ld}}, M_{\mathrm{td}}$, and $M_{\mathrm{rd}}$ are the $3 \mathrm{D}$ components of the vector $\mathbf{M}$ in the lamination coordinate system, and $M_{x}, M_{y}$, and $M_{z}$ are the $3 \mathrm{D}$ components of the vector $\mathbf{M}$ in the $3 \mathrm{D}$ space rectangular coordinate system in equations (1) to (5).

2.2. Vibration Analysis Method. The vibration of the core is caused by the Maxwell force and magnetostriction in the magnetic field, which are affected by the spatial distribution of the magnetic field.

In the spatial rectangular coordinate system, the $3 \mathrm{D}$ electromagnetic equation of the reactor core under normal operating conditions is given by 


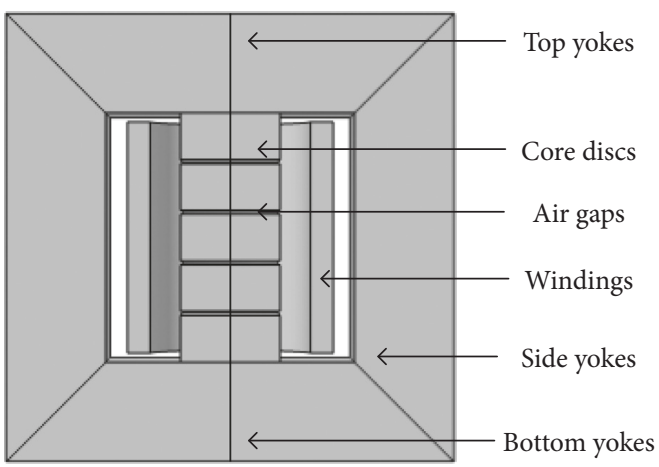

(a)

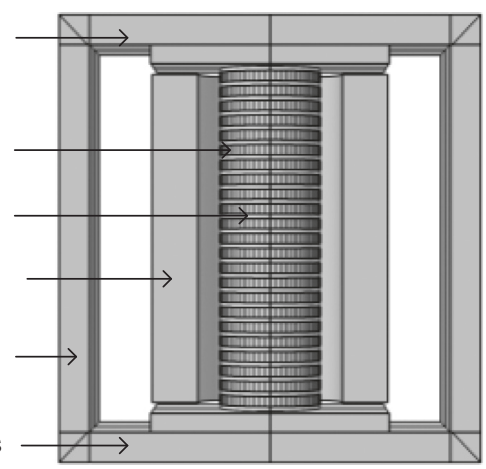

(b)

Figure 1: Simplified diagrams of two types of reactors: (a) small dry reactor and (b) large oil-immersed shunt reactor.

TABLE 1: The mapping between the two coordinate systems.

\begin{tabular}{|c|c|c|c|c|}
\hline & Part & $\mathrm{RD}$ & $\mathrm{TD}$ & LD \\
\hline Large oil-immersed shunt reactors & $\begin{array}{c}\text { Core disc } \\
\text { Top and bottom yokes } \\
\text { Side yokes }\end{array}$ & $\begin{array}{l}z \text {-axis } \\
y \text {-axis } \\
z \text {-axis }\end{array}$ & $\begin{array}{l}\text { Vertical to radial and } z \text {-axis } \\
\begin{array}{c}z \text {-axis } \\
y \text {-axis }\end{array}\end{array}$ & $\begin{array}{l}x \text { Radial } \\
x \text {-axis } \\
x \text {-axis }\end{array}$ \\
\hline Small dry reactors & $\begin{array}{l}\text { Core discs } \\
\text { Yokes }\end{array}$ & $\begin{array}{l}y \text {-axis } \\
x \text {-axis }\end{array}$ & $\begin{array}{l}x \text {-axis } \\
y \text {-axis }\end{array}$ & $\begin{array}{l}z \text {-axis } \\
z \text {-axis }\end{array}$ \\
\hline
\end{tabular}

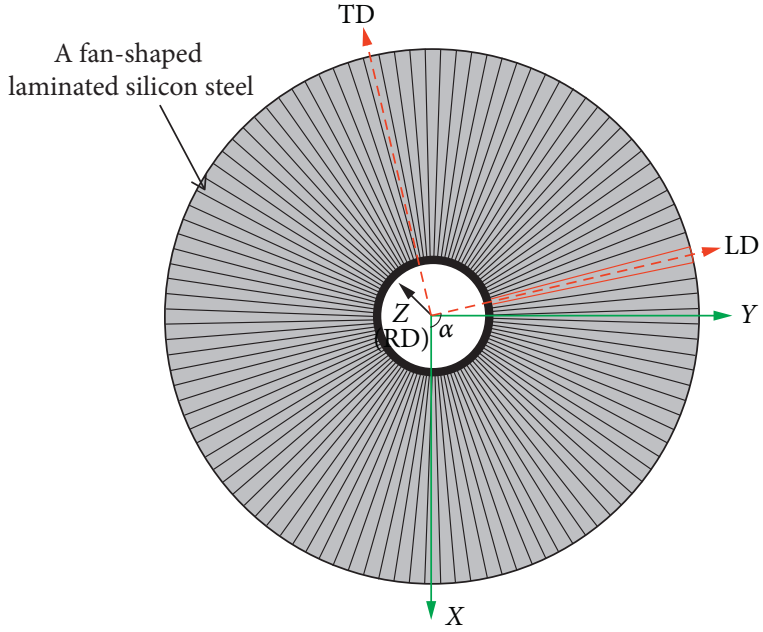

Figure 2: A plan view of a core disc: the green arrow is the coordinate axis of the space rectangular coordinate system, and the red arrow represents the coordinate axis of the laminated coordinate system.

$$
\left\{\begin{array}{c}
\frac{\partial}{\partial y}\left[v_{z}\left(\frac{\partial A_{y}}{\partial x}-\frac{\partial A_{x}}{\partial y}\right)\right]-\frac{\partial}{\partial z}\left[v_{y}\left(\frac{\partial A_{x}}{\partial z}-\frac{\partial A_{z}}{\partial x}\right)\right]=J_{x} \\
\frac{\partial}{\partial z}\left[v_{x}\left(\frac{\partial A_{z}}{\partial y}-\frac{\partial A_{y}}{\partial z}\right)\right]-\frac{\partial}{\partial x}\left[v_{z}\left(\frac{\partial A_{y}}{\partial x}-\frac{\partial A_{x}}{\partial y}\right)\right]=J_{y}, \\
\frac{\partial}{\partial x}\left[v_{y}\left(\frac{\partial A_{x}}{\partial z}-\frac{\partial A_{z}}{\partial x}\right)\right]-\frac{\partial}{\partial y}\left[v_{x}\left(\frac{\partial A_{z}}{\partial y}-\frac{\partial A_{y}}{\partial z}\right)\right]=J_{z}
\end{array}\right.
$$

where $v_{x}, v_{y}$, and $v_{z}$ are the components of the magnetoresistance ratio $v ; A_{x}, A_{y}$, and $A_{z}$ are the components of the vector magnetic potential $\mathbf{A}$; and $J_{x}, J_{y}$, and $J_{z}$ are the components of the winding current density $\mathbf{J}$.

The magnetic flux density $\mathbf{B}$ in the spatial rectangular coordinate system can be calculated by

$$
\mathbf{B}=\nabla \times \mathbf{A} .
$$

The magnetic flux density $\mathbf{B}$ in the lamination coordinate system was obtained through coordinate transformation (equations (1) to (5)) of $\mathbf{B}$ in the spatial rectangular coordinate system. The magnetic field strength $\mathbf{H}$ was calculated by the 3D electromagnetic field constitutive relationship as follows:

$$
\mathbf{H}=\left[\begin{array}{c}
H_{\mathrm{rd}} \\
H_{\mathrm{td}} \\
H_{\mathrm{ld}}
\end{array}\right]=\left[\begin{array}{ccc}
v_{\mathrm{rd}} & 0 & 0 \\
0 & v_{\mathrm{td}} & 0 \\
0 & 0 & v_{\mathrm{ld}}
\end{array}\right]\left[\begin{array}{c}
B_{\mathrm{rd}} \\
B_{\mathrm{td}} \\
B_{\mathrm{ld}}
\end{array}\right] .
$$

The 3D magnetisation curves ( $\mathbf{H}-\mathbf{B}$ curves, where $\mathbf{H}$ is the magnetic field strength in the lamination coordinate system and $\mathbf{H}$ is the magnetic flux density in the laminated coordinate system) of a type of laminated silicon steel were obtained by tests [10] (Figure 3).

The Maxwell force $\mathbf{F}_{\max }$ acting on the interfaces between core discs and air gaps in the magnetic field can be obtained from equation (9) [11-13]:

$$
\mathbf{F}_{\max }=\int_{s} \mathbf{T}_{\max } \mathrm{d} S
$$

where $\mathbf{T}_{\max }$ is the Maxwell stress tensor (equation (11)).

The magnetostriction phenomenon is caused by the displacement of the magnetic domain inside the core (silicon steel) in the magnetic field, and its magnitude is closely related to the magnetic field. Magnetostrictive displacement and strain are obtained from the magnetic field strength by 


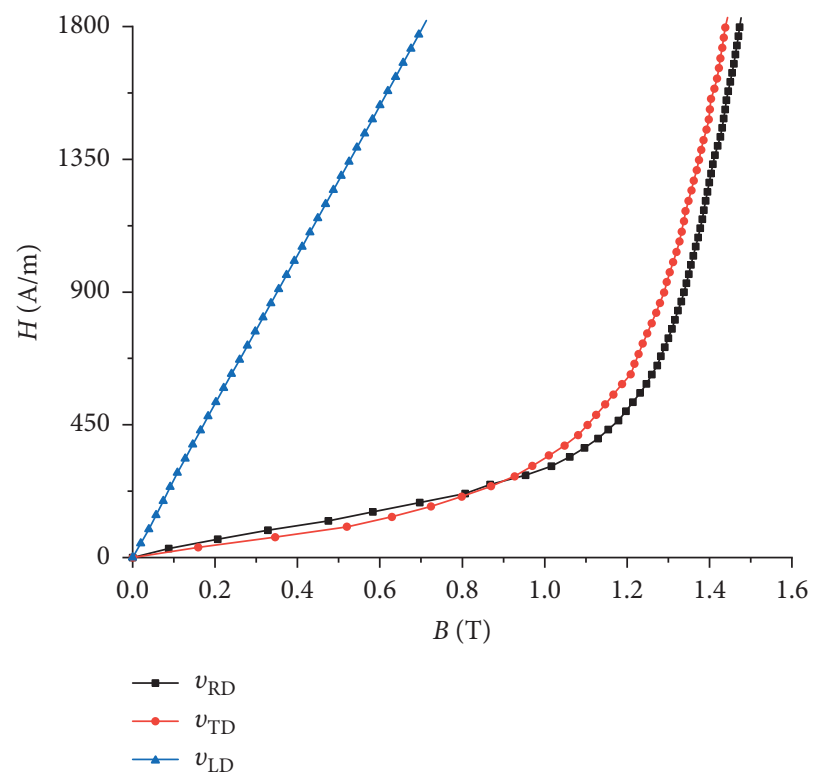

Figure 3: The 3D magnetisation curves (H-B curves) of the laminated silicon steel.

the magnetostrictive curves of the laminated silicon steel. The generalised form of Hooke's law (equation (10)) can be used to describe the relationship between elastic stress and strain.

$$
\begin{aligned}
& \boldsymbol{\sigma}_{e}=\mathbf{D} \boldsymbol{\varepsilon}_{e}, \\
& \mathbf{T}_{\max }=\left[\begin{array}{ccc}
B_{\mathrm{rd}} H_{\mathrm{rd}}-\frac{1}{2} B H & B_{\mathrm{rd}} H_{\mathrm{td}} & B_{\mathrm{rd}} H_{\mathrm{ld}} \\
B_{\mathrm{td}} H_{\mathrm{rd}} & B_{\mathrm{td}} H_{\mathrm{td}}-\frac{1}{2} B H & B_{\mathrm{td}} H_{\mathrm{ld}} \\
B_{\mathrm{ld}} H_{\mathrm{rd}} & B_{\mathrm{ld}} H_{\mathrm{td}} & B_{\mathrm{ld}} H_{\mathrm{ld}}-\frac{1}{2} B H
\end{array}\right]\left[\begin{array}{c}
n_{\mathrm{rd}} \\
n_{\mathrm{td}} \\
n_{\mathrm{ld}}
\end{array}\right], \\
& \mathbf{D}=\frac{E(1-v)}{(1+v)(1-2 v)} \cdot\left[\begin{array}{cccccc}
1 & \frac{v}{1-v} & \frac{v}{1-v} & 0 & 0 & 0 \\
\frac{v}{1-v} & 1 & \frac{v}{1-v} & 0 & 0 & 0 \\
\frac{v}{1-v} & \frac{v}{1-v} & 1 & 0 & 0 & 0 \\
0 & 0 & 0 & \frac{1-2 v}{2(1-v)} & 0 & 0 \\
0 & 0 & 0 & 0 & \frac{1-2 v}{2(1-v)} & 0 \\
0 & 0 & 0 & 0 & 0 & \frac{1-2 v}{2(1-v)}
\end{array}\right] \text {, } \\
& \boldsymbol{\varepsilon}_{e}=\left[\begin{array}{c}
\varepsilon_{r d} \\
\varepsilon_{t d} \\
\varepsilon_{l d} \\
\gamma_{r t} \\
\gamma_{t l} \\
\gamma_{l r}
\end{array}\right]
\end{aligned}
$$


where $\mathbf{D}$ is the elastic tensor of core material (equation (12)) and $\varepsilon_{e}$ is the strain in the core material due to magnetostriction (equation (13)). $E$ and $v$ are Young's modulus and Poisson's ratio of the core material, respectively; $\varepsilon_{\mathrm{rd}}, \varepsilon_{\mathrm{td}}$, and $\varepsilon_{\mathrm{ld}}$ are the principal strains in the material; and $\gamma_{\mathrm{rt}}, \gamma_{\mathrm{rt}}$, and $\gamma_{\mathrm{tl}}$ are tangential strains. Regardless of the shear component, as in the following equation:

$$
\boldsymbol{\varepsilon}=\left[\begin{array}{c}
\varepsilon_{\mathrm{rd}} \\
\varepsilon_{\mathrm{td}} \\
\varepsilon_{\mathrm{ld}}
\end{array}\right]=\boldsymbol{\lambda}=\left[\begin{array}{c}
\lambda_{\mathrm{rd}} \\
\lambda_{\mathrm{td}} \\
\lambda_{\mathrm{ld}}
\end{array}\right],
$$

where

$$
\lambda=\left[\begin{array}{c}
\lambda_{\mathrm{rd}} \\
\lambda_{\mathrm{td}} \\
\lambda_{\mathrm{ld}}
\end{array}\right]=\left[\begin{array}{ccc}
k_{\mathrm{rd}} & 0 & 0 \\
0 & k_{\mathrm{td}} & 0 \\
0 & 0 & k_{\mathrm{ld}}
\end{array}\right]\left[\begin{array}{c}
B_{\mathrm{rd}} \\
B_{\mathrm{td}} \\
B_{\mathrm{ld}}
\end{array}\right] .
$$

$k_{\mathrm{rd}}$ and $k_{\mathrm{td}}$ are experimentally measured magnetostrictive coefficients of the silicon steel laminate in the RD and TD. The magnetostrictive curves of a type of nonoriented silicon steel were obtained by tests [8] (Figure 4). The magnetostriction coefficient of the LD is currently not available. The magnetostriction of silicon steel is an elastic deformation in which the total volume of the material remains constant [14, 15]. Hence,

$$
\left(1+\lambda_{\mathrm{rd}}\right)\left(1+\lambda_{\mathrm{td}}\right)\left(1+\lambda_{\mathrm{ld}}\right)=1 .
$$

The vibration differential equation of the reactor core is given by

$$
\mathbf{M} \frac{\partial^{2} \mathbf{u}}{\partial t^{2}}+\mathbf{C} \frac{\partial \mathbf{u}}{\partial t}+\mathbf{K u}=\mathbf{F}_{\text {total }},
$$

where $\mathbf{M}$ is the mass matrix of the core, $\mathbf{C}$ is the damping matrix, $\mathbf{K}$ is the stiffness matrix, $\mathbf{u}$ is the displacement vector, and $\mathbf{F}_{\text {total }}$ is the external force acted on the core, which is the resultant force of the Maxwell force and the magnetostrictive stress.

$$
\begin{aligned}
& \mathbf{F}_{\text {total }}=\mathbf{F}_{\text {max }}+\mathbf{F}_{\text {mag }}, \\
& \mathbf{F}_{\text {mag }}=-\nabla \cdot \boldsymbol{\sigma} .
\end{aligned}
$$

Regardless of the damping of the vibration process, equation (17) can be simplified to

$$
\mathbf{M} \frac{\partial^{2} \mathbf{u}}{\partial t^{2}}+\mathbf{K u}=\mathbf{F}_{\max }+\mathbf{F}_{\text {mag }} .
$$

\section{Comparison of 2D and 3D Methods}

2D methods have been widely used to study the vibration characteristics of small dry reactor cores, but the new 3D method also has a wide range of applications. Two sets of comparative studies were conducted to compare the differences between the two methods in analysing the vibration of small dry reactors and large oil-immersed shunt reactors. The magnetic flux density (B) and acceleration (ac) of the core vibration were selected as comparison indicators. The

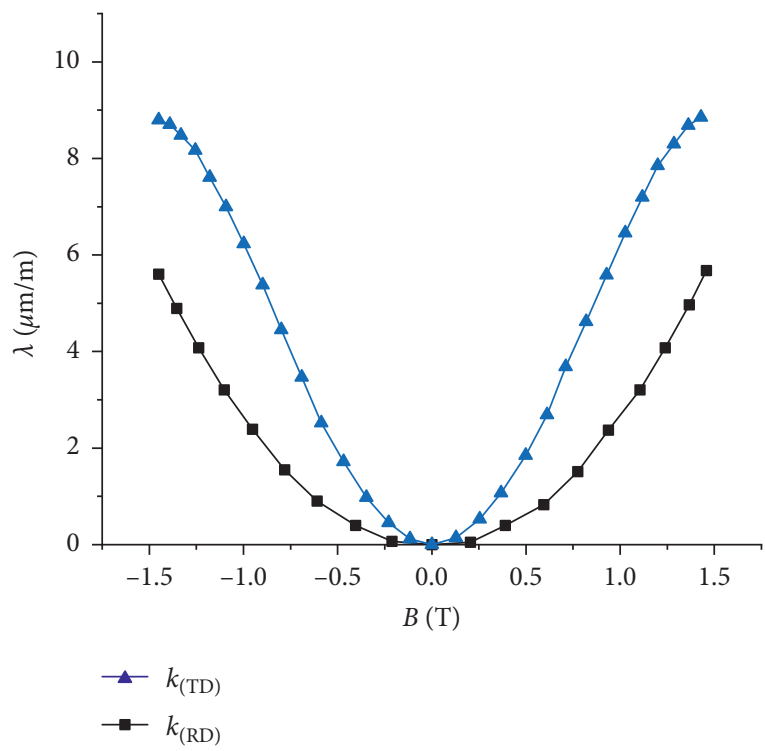

Figure 4: The magnetostrictive curves of the laminated silicon steel.

difference rate $(\eta)$ was defined and used to compare the size of the difference between the two methods, as given by

$$
\eta_{X}=\left|\frac{X_{2 \mathrm{D}}-X_{3 \mathrm{D}}}{X_{3 \mathrm{D}}}\right| \times 100 \%,
$$

where $X$ refers to two contrast indicators (B and ac).

In particular, the $2 \mathrm{D}$ method used here stems from other work $[3,8]$. The parameters of reactors are provided by China TBEA Hengyang Transformer Co., Ltd and China Xi'an Xidian Transformer Co., Ltd (Table 2). The bottom of the reactor is generally connected to the ground and is set as a fixed boundary.

The $2 \mathrm{D}$ and $3 \mathrm{D}$ methods were used separately, and the differences therein (Table 3) were obtained. In terms of the calculation results of the small dry reactor, the distributions (Figure 5) of the two sets of comparative indicators obtained by the two methods are similar. The difference $(4.5 \%$ and $10.8 \%$ ) of the average of the two sets of comparative indicators is also practicable (Table 3). Therefore, the two methods are mutually replaceable for the study of small dry reactors. An experiment on the core vibration of a small dry reactor was carried out in reference [3], which verified the $2 \mathrm{D}$ method. The difference between the 3D method given here and the 2D method is small for the vibration parameters of the small dry reactor. Therefore, the calculation result of the $3 \mathrm{D}$ method proposed here is also correct; however, the 2D method and the 3D method are not equivalent for the large oil-immersed shunt reactors (Figure 6) because the differences $(33.1 \%$ and $154.14 \%)$ in the two methods are too large to be ignored (Table 3). Compared with the 2D method, the 3D method yields the same analytical results for small dry reactors and is more accurate for large oil-immersed reactors. Therefore, the new $3 \mathrm{D}$ method should be adopted instead of the traditional 2D method for the large oil-immersed shunt reactors. 
TABLe 2: Parameters of two types of reactors.

\begin{tabular}{lcc}
\hline Parameter & Large oil-immersed shunt reactor & Small dry reactor \\
\hline Length of iron yoke $(\mathrm{mm})$ & 2980 & 270 \\
Width of iron yoke $(\mathrm{mm})$ & 3045 & 60 \\
Thickness of iron yoke (mm) & 1460 & 78 \\
Height of winding (mm) & 2650 & 142 \\
Width of winding (mm) & 372 & 14 \\
Radius of winding (mm) & 955 & 97.58 \\
Height of core limb (mm) & 2547 & 150 \\
Winding turns & 2147 & 10 \\
Density of the core $\left(\mathrm{kg} / \mathrm{m}^{3}\right)$ & 7650 & 7650 \\
Young's modulus of the core $(\mathrm{GPa})$ & 195 & 195 \\
Poisson's ratio of the core & 0.27 & 0.27 \\
Young's modulus of gaps $(\mathrm{GPa})$ & 55 & 55 \\
Poisson's ratio of gaps & 0.45 & 0.45 \\
Height of an air gap (mm) & 38 & 28 \\
Number of air gaps & 24 & 4 \\
Height of a cake (mm) & 80 & 78 \\
Radius of core limb (mm) & 405 & 1 \\
Width of core limb (mm) & 1 & 60 \\
Simulation condition & Frequency domain \\
Rated current (A) & 377.9 & 300 \\
Current frequency (Hz) & 50 & 50 \\
\hline
\end{tabular}

TABLE 3: The comparison of $2 \mathrm{D}$ and 3D methods.

\begin{tabular}{lccc}
\hline & & $\mathrm{B}(\mathrm{T})$ & $\mathrm{ac}\left(\mathrm{m} / \mathrm{s}^{2}\right)$ \\
\hline \multirow{3}{*}{ Small dry reactor } & $2 \mathrm{D}$ & 0.21 & $2.96 \times 10^{-3}$ \\
& 3D & 0.22 & $3.32 \times 10^{-3}$ \\
& $\eta(\%)$ & 4.5 & 10.8 \\
\hline \multirow{3}{*}{ Large oil-immersed shunt reactor } & $2 \mathrm{D}$ & 1.57 & 16.57 \\
& 3D & 1.18 & 6.52 \\
& $\eta(\%)$ & 33.1 & 154.14 \\
\hline
\end{tabular}

\section{Influence of Air Gap Number on UHV Shunt Reactor Core Vibration}

Since 2006, China has established several UHV (1100kV) transmission lines for long-distance, large-capacity transmission [16]. A UHV shunt reactor is an important item of equipment in UHV AC transmission projects. Excessive vibration is one of the most common problems in the operation thereof due to the air gaps in iron beam that provide large inductance and the large rated capacity, which is seven to eight times that of a $500 \mathrm{kV}$ reactor [17]. Adjusting the air gaps can be a useful method to reduce vibrations therein. The influence of air gap number on core vibration of the UHV shunt reactor is of interest here. A UHV shunt reactor is a typical oil-immersed shunt reactor, so the new 3D method was adopted.

The number of air gap is not allowed to vary widely because of other performance requirements of large oilimmersed shunt reactors. So, the number of air gaps $(g)$ in the core limb is set to $20,22,24,26$, and 28 . The number of air gaps affects the inductance of the reactor, which is one of the most important parameters of the reactor. The total length of the air gap is adjusted so that the inductance values remain the constant. The calculation method of the reactor inductance is from the reference [18], and the corresponding relationship between the number of air gaps and the total length of the air gap is shown in Table 4 . The other reactor parameters are as listed in Table 2.

4.1. Results of UHV Shunt Reactor Core Vibration. The displacement $(d)$ and acceleration (ac) at each point of the core during its vibration were selected to characterise the vibration strength of the reactor core according to equation (19).

The method of 3D finite element simulation was used, and the distribution map of $d$ was obtained, which shows a gradual increase with increasing number of air gaps (Figure 7). The average displacement of the core, the iron yoke, and the core disc with different air gap numbers was also calculated (Figure 8). They all show increase with increasing air gap numbers. Taking the average value of the core as an example, $d=4.58 \times 10^{-2} \mathrm{~mm}$ when the air gap number is 20 and when $g=24, d=6.06 \times 10^{-2} \mathrm{~mm}$, which is approximately 1.5 times the value at $g=20$, the maximum value of $d$ is $8.77 \times 10^{-2} \mathrm{~mm}$, at $g=28$. The variation of the mean average value of $d$ is similar in the iron yoke and the core disc.

The distribution of vibration acceleration and the average value were also calculated with different air gap numbers (Figures 9 and 10). The trend in acceleration is similar to that in the displacement (i.e., both increase).

Both the displacement and the acceleration of the core vibration increase with the increase in the air gap number. It can be concluded that the increase in the air gap number will aggravate the vibration of the reactor core while maintaining the overall size (length, width, and height) and inductance of 


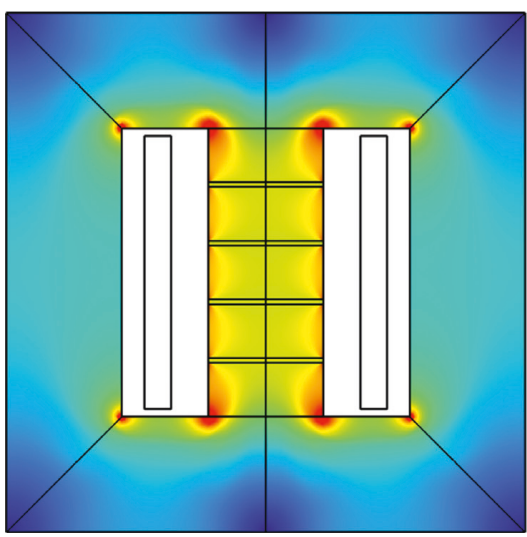

B-2D

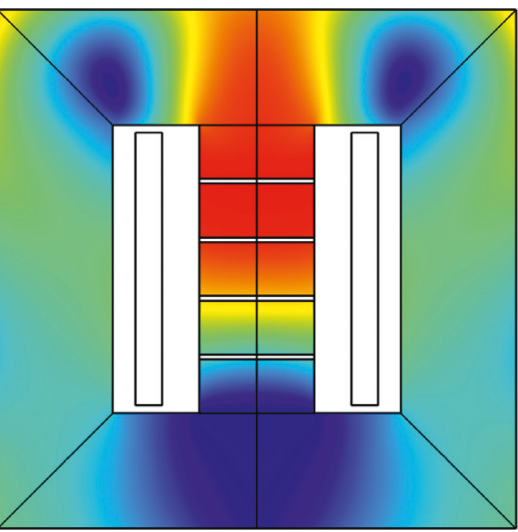

ac-2D

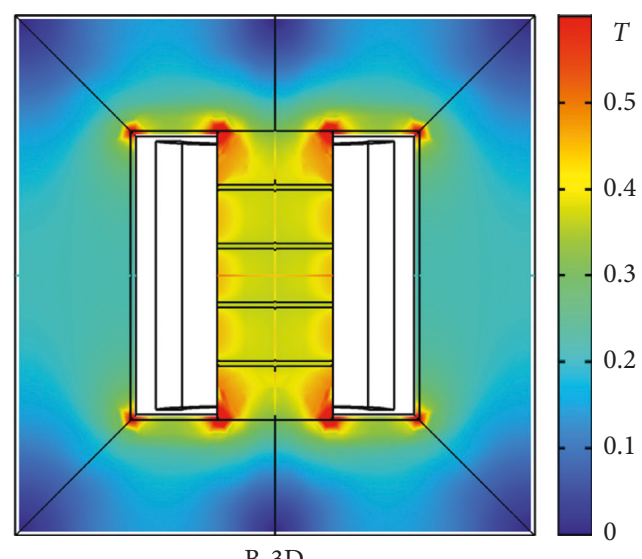

B-3D

(a)

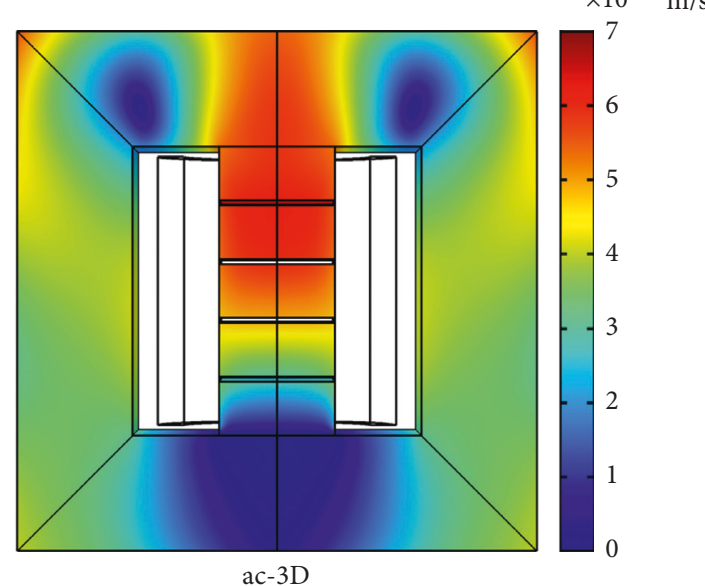

(b)

FIgure 5: The distributions of comparative indicators for small dry reactor. (a) The magnetic flux density. (b) The acceleration. The distributions of magnetic flux density and vibration acceleration of the small dry reactor core obtained by the two calculation methods are similar.

the core. In the future reactor design, the minimum number of core air gaps should be chosen to reduce core vibration.

\subsection{Discussion of UHV Shunt Reactor Core Vibration.} The stress on the core is the main cause of the vibration of the reactor core. The acceleration and displacement of the core vibration are positively related to the stress on it. Defining the average value of stress $\left(\boldsymbol{\sigma}_{\text {average }}\right)$ on the core as

$$
\boldsymbol{\sigma}_{\text {average }}=\frac{\mathbf{F}_{\text {total }}}{S_{\text {total }}}=\frac{\int \boldsymbol{\sigma}_{\text {total }} \mathrm{d} s}{S_{\text {total }}},
$$

where $S$ is total surface area of the core.

$\boldsymbol{\sigma}_{\text {average }}$ on the core was calculated as shown in Figure 11. It increases with the increase in the number of air gaps, which causes the increase in core vibration indicators.

Magnetic flux density is one of the important factors affecting the stress on the core in the magnetic field. The distribution and average value of magnetic flux density in the reactor with different air gap numbers are as shown in Figures 12 and 13. Magnetic flux density increases slightly with increasing air gap numbers. The inductance of the reactor decreases with the increase of the number of air gaps, on the premise that the magnetic flux density and the total length of air gap remain unchanged [18]. Here, the total length of air gap is reduced to maintain the inductance of the reactor at a constant level, when the number of air gaps increases.

The magnetic flux can be divided into two parts: the main magnetic flux and the leakage magnetic flux. The material in the air gap of a reactor is generally marble or a ceramic. The permeability of these two materials is much lower than that of silicon steel, which is an important reason for the observed magnetic flux leakage. The leakage of reactor core is reduced when the length of air gap decreases, so the average magnetic flux density increases with the increase of air gap number.

The force on the core is the resultant force of the Maxwell force on the core discs and the magnetostrictive force on the core. The Maxwell force is greater than the magnetostrictive force [9]. The Maxwell force is only applied to the core discs, and it is a surface stress that is related to the unit area stress 


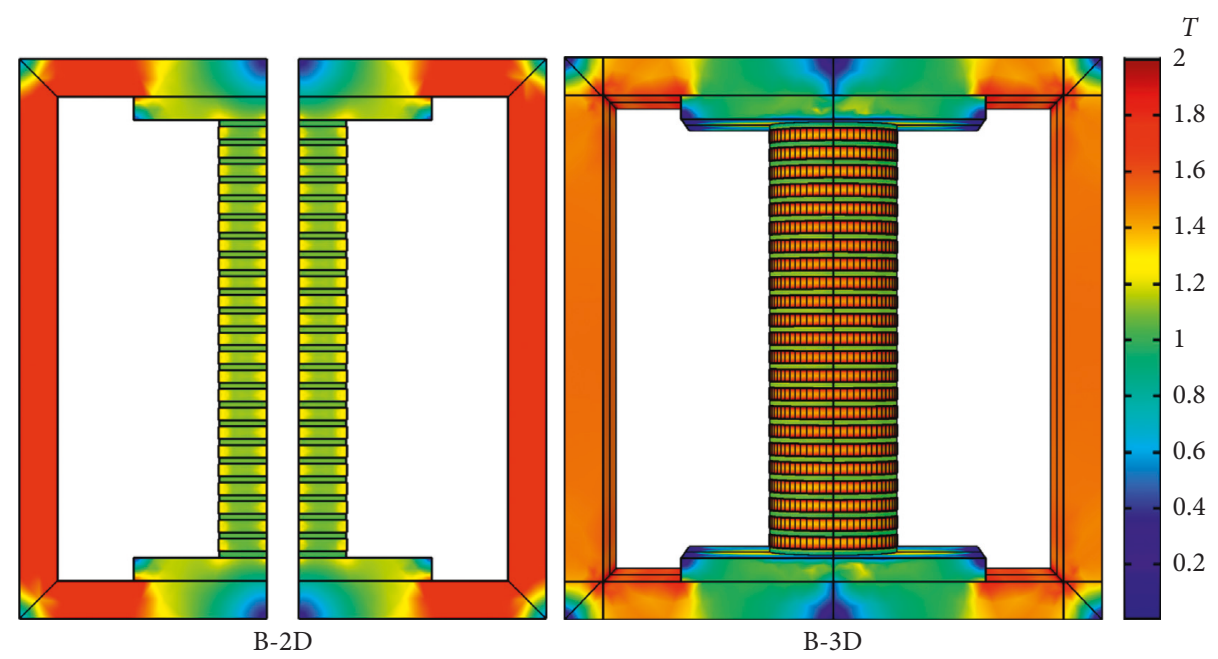

(a)

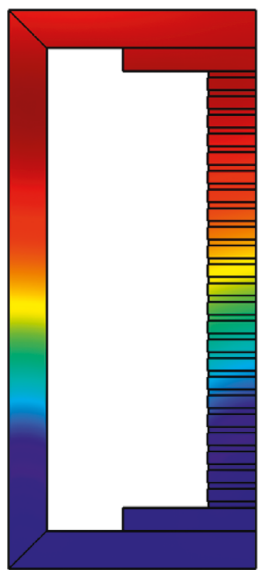

$\mathrm{ac}-2 \mathrm{D}$

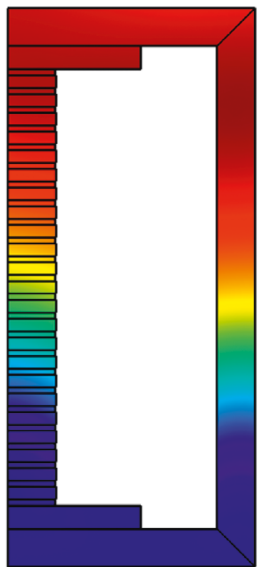

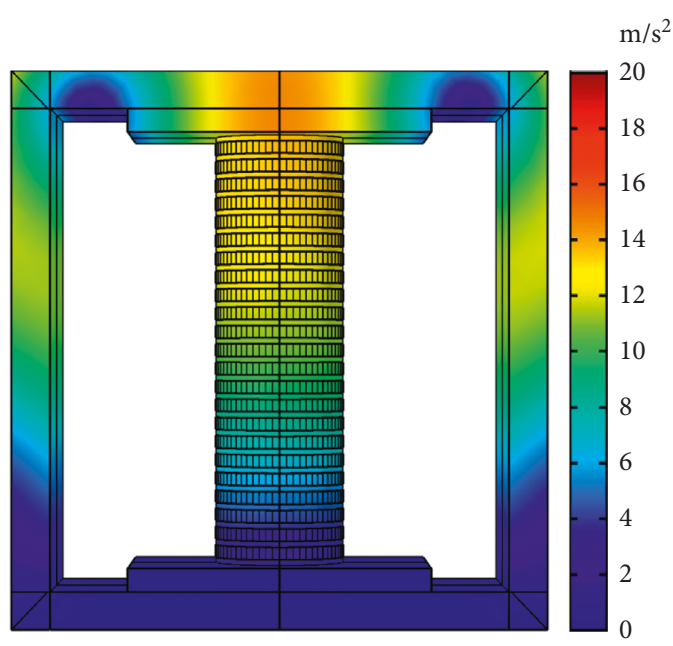

ac-3D

(b)

Figure 6: The distributions of comparison indicators for large oil-immersed shunt reactor. (a) The magnetic flux density. (b) The acceleration. The distributions of magnetic flux density and vibration acceleration of the large oil-immersed reactor core obtained by the two calculation methods are very different.

TABLE 4: Total length of air gap with different $g$.

\begin{tabular}{llllll}
\hline$g$ & 20 & 22 & 24 & 26 & 28 \\
$d(\mathrm{~mm})$ & 952.52 & 816.18 & 707 & 628.75 & 566.82 \\
\hline
\end{tabular}

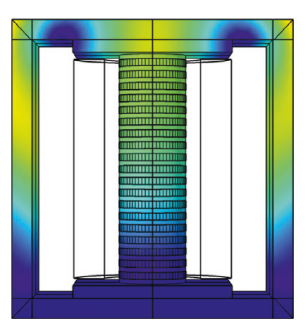

$g=20$

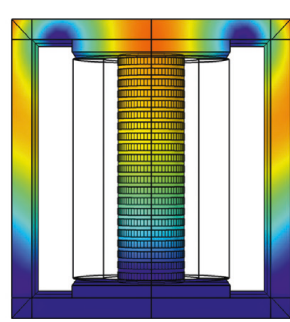

$g=22$

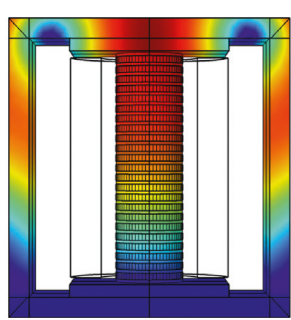

$g=24$

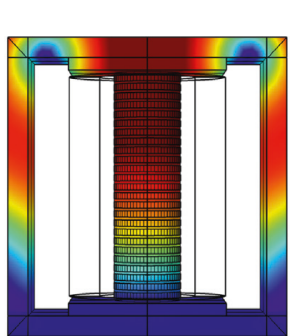

$g=26$

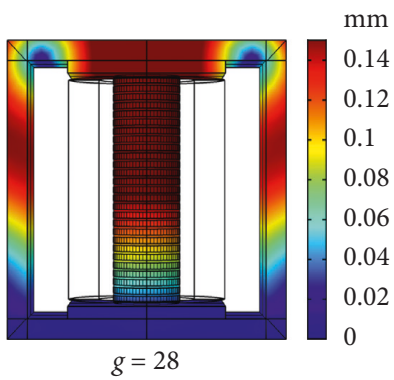

FIGURE 7: The distribution of vibration displacement $(d)$ with different air gap numbers. The vibration displacement of the reactor core increases as the number of air gaps increases. 


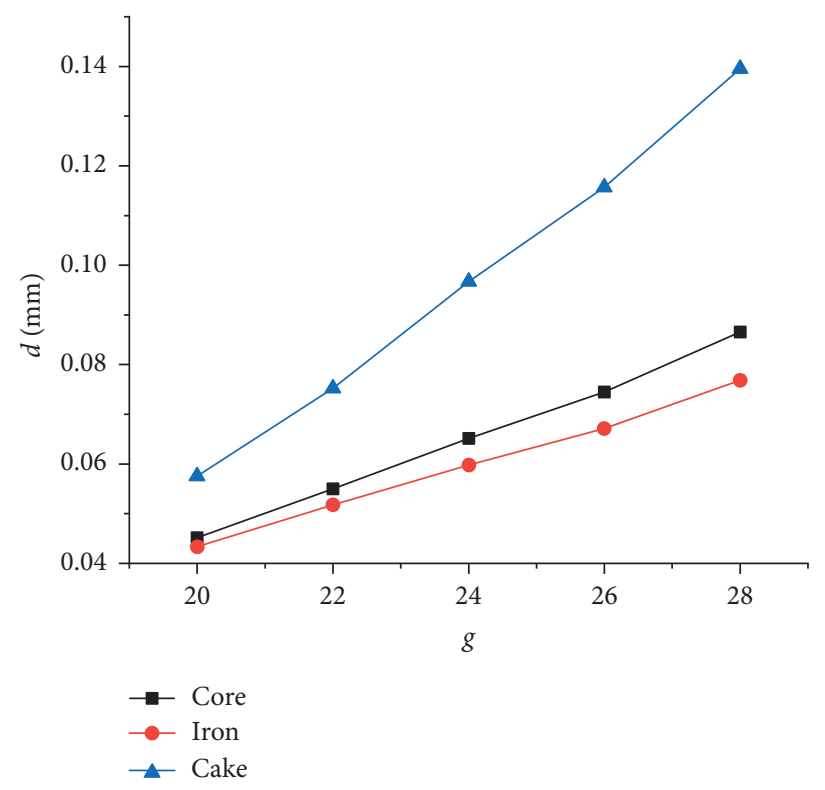

Figure 8: The average of displacement $(d)$ with different air gap numbers. The vibration displacement of each component (iron and cakes) of the reactor core increases as the number of air gaps increases.

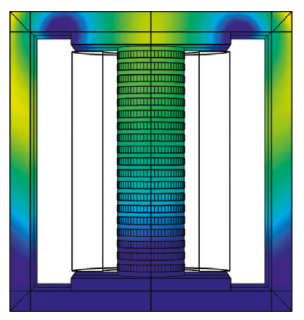

$g=20$

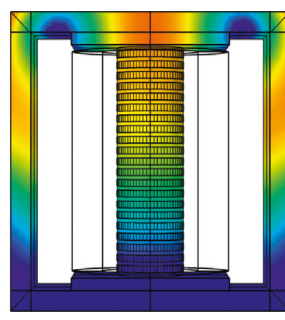

$g=22$

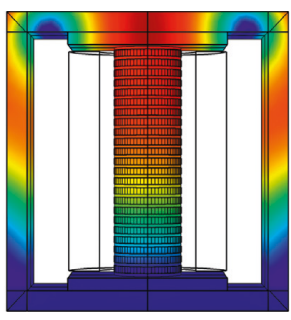

$g=24$

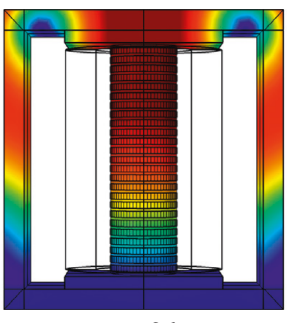

$g=26$

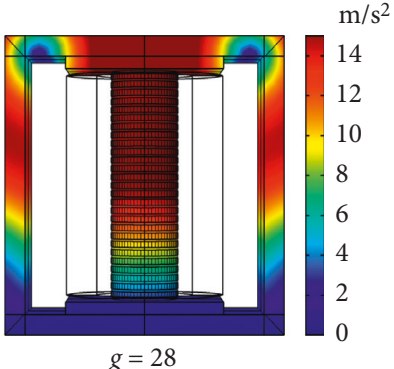

$g=28$

Figure 9: The distribution of vibration acceleration (ac) with different air gap numbers. The vibration acceleration of the reactor core increases as the number of air gaps increases.

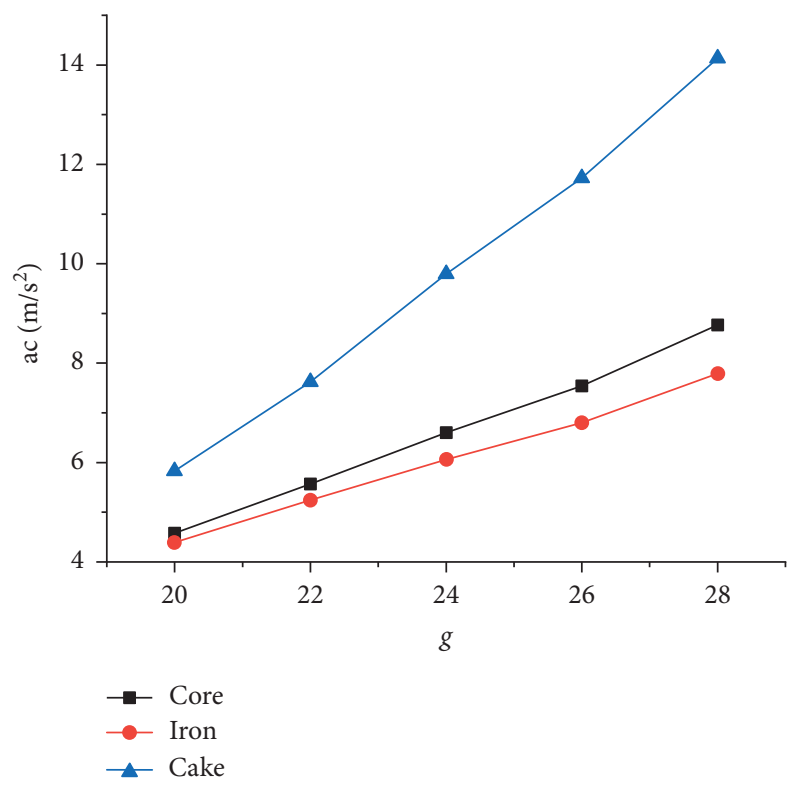

FIGURE 10: The average of acceleration (ac) with different air gap numbers. The vibration acceleration of each component (iron and cakes) of the reactor core increases as the number of air gaps increases. 


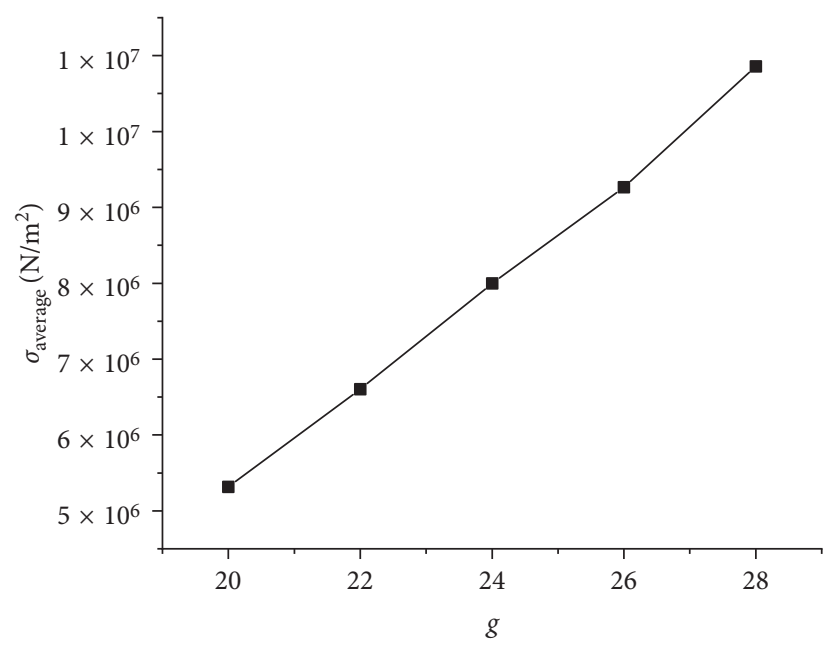

FIGURE 11: $\boldsymbol{\sigma}_{\text {average }}$ on the core with different air gap numbers. The average value of stress $\left(\boldsymbol{\sigma}_{\text {average }}\right)$ increases as the number of air gaps increases.
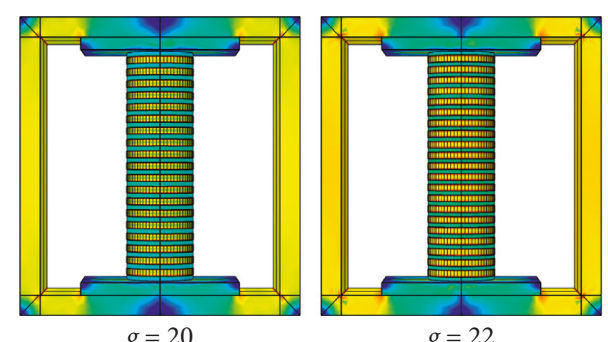

$g=22$

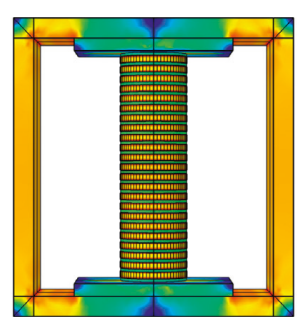

$g=24$

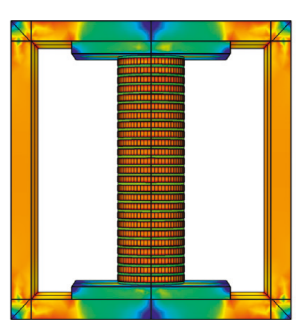

$g=26$

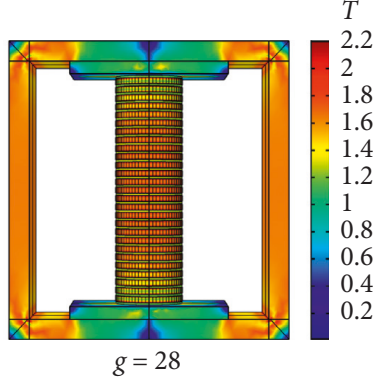

Figure 12: The distribution of flux density $(B)$ with different air gap numbers. The magnetic flux density of the core increases as the number of air gaps increases.

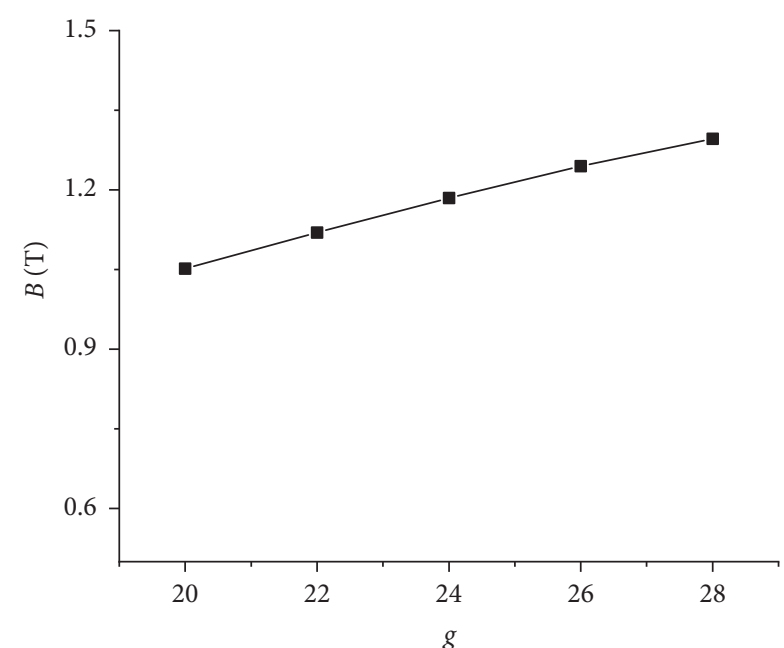

Figure 13: The average value of flux density $(B)$ with different air gap numbers. The average value of the magnetic flux density increases as the number of air gaps increases.

and the total area acted upon by the applied force. The Maxwell force per unit area is positively correlated with the magnetic flux density. The total stress area of the core and the magnetic flux density both increase with the increase in the air gaps. So, the total Maxwell force increases as the number of air gaps increases. The magnetostrictive force can be calculated by the magnetic flux density and the magnetostriction coefficient, and the relationship between the magnetostriction coefficient and the magnetic flux density is shown in Figure 4. The magnetostrictive force also increases due to the increase in magnetic flux density. Therefore, the increase in stress on the core is attributed to the increase in magnetic flux density and the total force area upon which the Maxwell force acts.

\section{Conclusion}

A new three-dimensional (3D) analysis method is proposed. First, a set of 3D laminated coordinate systems was defined based on silicon steel lamination rules, in which the anisotropy of magnetic properties for laminated silicon steel in the rolling direction (RD), the transverse direction (TD), and the lamination direction (LD) were considered. Then, the mapping between laminated coordinate systems and space rectangular coordinate system was established to unify the parameters in different laminated coordinate systems. After mapping, the anisotropy of the magnetic properties in the laminated coordinate systems was transformed into a spatial rectangular coordinate system. 
The 3D method was compared with the traditional 2D method by two sets of comparative studies. Applied to small dry reactors, the two methods differ by less than $10.8 \%$. The two methods are mutually replaceable for the study of small dry reactors. The difference is greater than $33.1 \%$ when they are used for large oil-immersed shunt reactors, so the new $3 \mathrm{D}$ method should be adopted instead of the traditional 2D method for large oil-immersed shunt reactors.

The 3D method was used for a UHV shunt reactor, which is a type of a large oil-immersed shunt reactor. On the premise that the inductance of the reactor is constant, the increase in core air gap number will increase the displacement and acceleration of the core vibration. With the increase of the air gap number in the reactor, the flux density in the core increases to keep the inductance of the reactor constant. The total force area upon which the Maxwell force on the core acts also increases with increasing air gap number. They are the two reasons for the intensification of core vibration. To reduce the vibration of the reactor core, the minimum number of air gaps should be selected in the design of the reactor core.

\section{Data Availability}

The data used to support the findings of this study are available from the corresponding author upon request.

\section{Conflicts of Interest}

The authors declare that they have no conflicts of interest.

\section{Acknowledgments}

This work was financially supported by the Fundamental Research Funds for the Central Universities of China (grant no.2019QN126).

\section{References}

[1] B. Tong, Y. Qingxin, Y. Rongge, and Z. Lihua, "Magnetically controlled saturable reactor core vibration under practical working conditions," IEEE Transactions on Magnetics, vol. 53, no. 6, pp. 1-4, 2017.

[2] P. Zhang and L. Li, "Vibration properties of two-stage magnetic-valve controllable reactor," IEEE Transactions on Magnetics, vol. 54, no. 3, pp. 1-4, 2018.

[3] F. Zhang, S. Ji, Y. Shi, and L. Zhu, "Investigation on the action of eddy current on tank vibration characteristics in dry-type transformer," IEEE Transactions on Magnetics, vol. 55, no. 2, pp. 1-8, 2019.

[4] Y. Gao, K. Muramatsu, M. J. Hatim et al., "Design of a reactor driven by inverter power supply to reduce the noise considering electromagnetism and magnetostriction," IEEE Transactions on Magnetics, vol. 46, no. 6, pp. 2179-2182, 2010.

[5] R. Yan, L. Zhao, T. Ben, and L. Zhu, "Design of lower vibration reactor," in Proceedings of the 2017 20th International Conference on Electrical Machines and Systems (ICEMS), pp. 1-5, Sydney, Australia, August 2017.

[6] M. Rossi and J. Le Besnerais, "Vibration reduction of inductors under magnetostrictive and Maxwell forces excitation," IEEE Transactions on Magnetics, vol. 51, no. 12, pp. 1-6, 2015.
[7] R. Yan, X. Gao, L. Zhu et al., "Research on three-dimensional stress distribution of reactor core," IEEE Transactions on Applied Superconductivity, vol. 26, no. 4, pp. 1-4, 2016.

[8] B. Tong, Y. Qingxin, Y. Rongge, Z. Lihua, and C. Junjie, "Stress analysis of inverter-fed induction motor considering anisotropic magnetization and magnetostrictive properties," IEEE Transactions on Applied Superconductivity, vol. 28, no. 3, pp. 1-4, 2018.

[9] Y. Gao, M. Nagata, K. Muramatsu, K. Fujiwara, Y. Ishihara, and S. Fukuchi, "Noise reduction of a three-phase reactor by optimization of gaps between cores considering electromagnetism and magnetostriction," IEEE Transactions on Magnetics, vol. 47, no. 10, pp. 2772-2775, 2011.

[10] L. Zhu, "Study of affection from magnetostriction in laminated core on vibration noise of transformer and AC motor," Hebei University of Technology, Tianjin, China, Dissertation, 2013.

[11] X. Xu, Q. Han, and F. Chu, "Review of electromagnetic vibration in electrical machines," Energies, vol. 11, no. 7, p. 1779, 2018.

[12] G. Zhang, J. Wu, and L. Hao, "Fast calculation model and theoretical analysis of rotor unbalanced magnetic pull for inter-turn short circuit of field windings of non-salient pole generators," Energies, vol. 10, no. 5, Article ID 732, 2017.

[13] Y. Chen, Y. Yang, and Y. Shen, "Influence of small teeth on vibration for dual-redundancy permanent magnet synchronous motor," Energies, vol. 11, no. 9, Article ID 2462, 2018.

[14] Y. Gao, K. Muramatsu, K. Fujiwara, Y. Ishihara, S. Fukuchi, and T. Takahata, "Vibration analysis of a reactor driven by an inverter power supply considering electromagnetism and magnetostriction," IEEE Transactions on Magnetics, vol. 45, no. 10, pp. 4789-4792, 2009.

[15] J. Gołdasz, B. Sapinski, and L. Jastrzębski, "Assessment of the magnetic hysteretic behaviour of MR dampers through sensorless measurements," Shock and Vibration, vol. 2018, Article ID 3740208, 21 pages, 2018.

[16] H. Zhao and S. Guo, "Risk evaluation on UHV power transmission construction project based on AHP and FCE method," Mathematical Problems in Engineering, vol. 2014, Article ID 687568, 14 pages, 2014.

[17] B. Han, L. Ban, Z. Xiang et al., "Analysis on strategies of suppressing secondary arc current in UHV system with controllable shunt reactors," in Proceedings of the 2016 IEEE International Conference on Power System Technology (POWERCON), pp. 1-5, Wollongong, Australia, SeptemberOctober 2016.

[18] A. Lotfi and E. Rahimpour, "Optimum design of core blocks and analyzing the fringing effect in shunt reactors with distributed gapped-core," Electric Power Systems Research, vol. 101, pp. 63-70, 2013. 


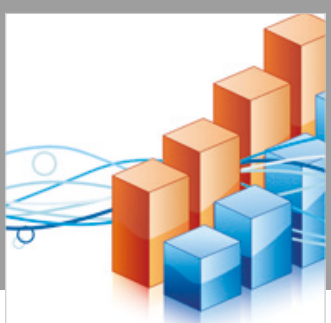

Advances in

Operations Research

\section{-n-m}
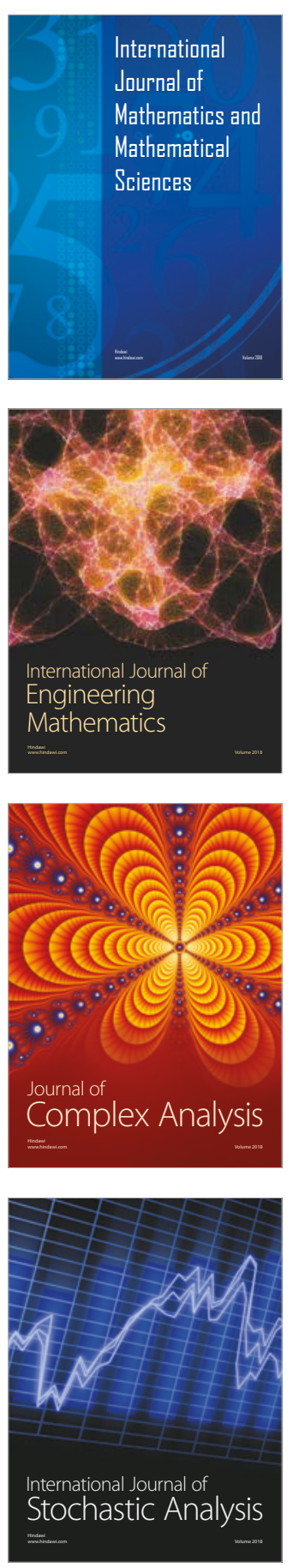
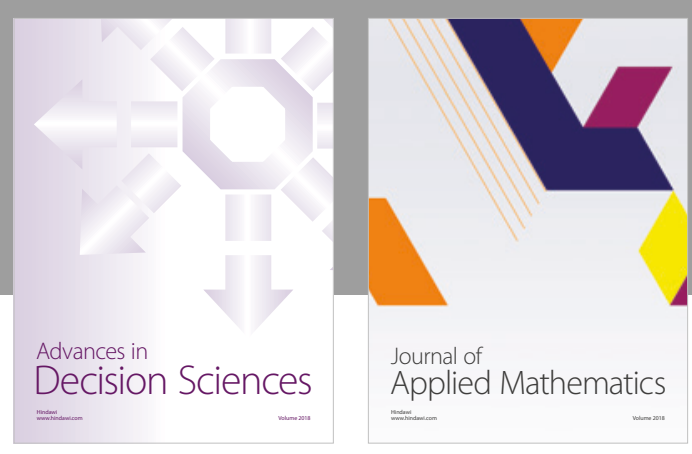

Journal of

Applied Mathematics
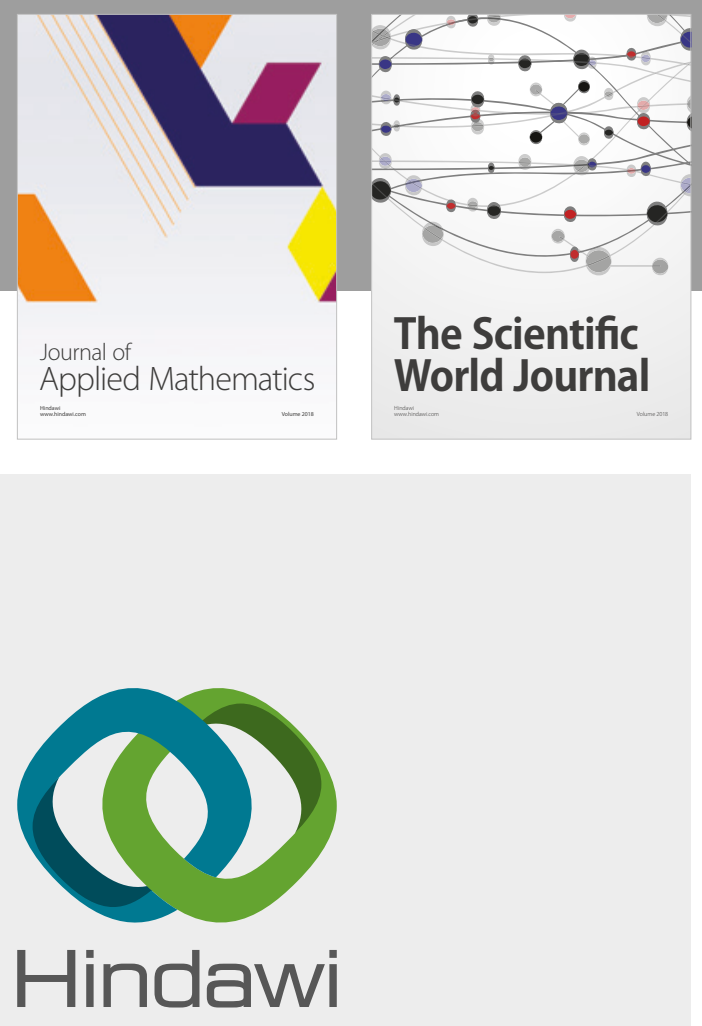

Submit your manuscripts at

www.hindawi.com

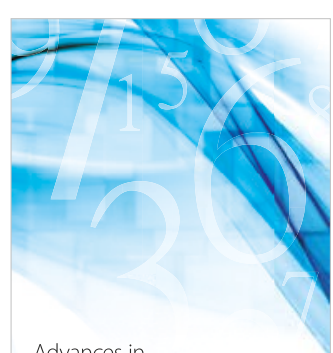

Advances in
Numerical Analysis
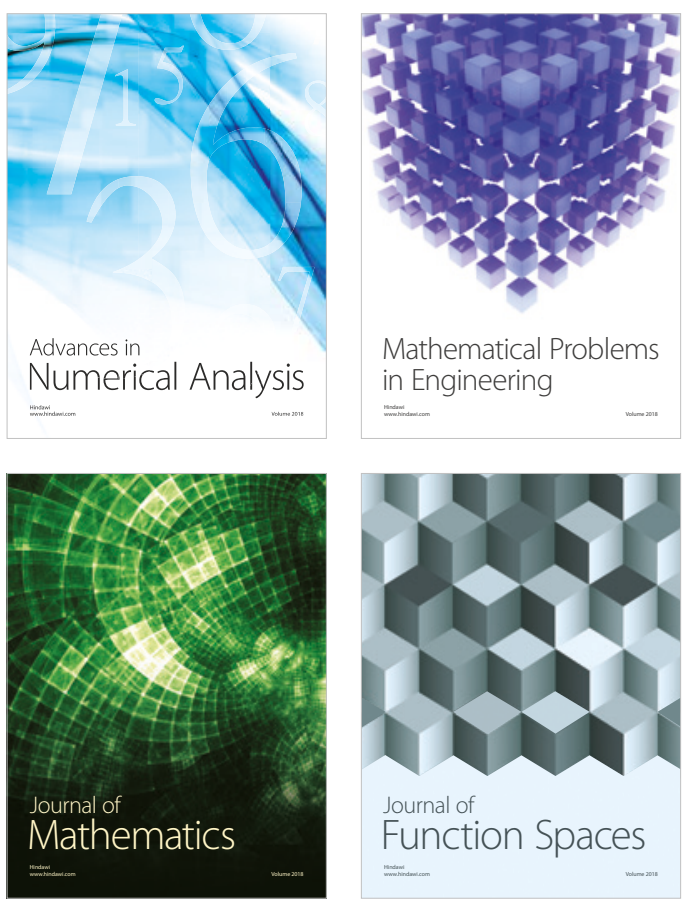

Mathematical Problems in Engineering

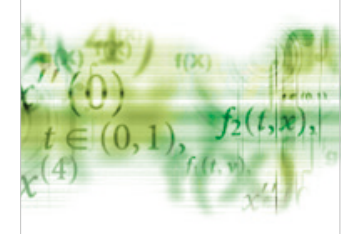

International Journal of

Differential Equations

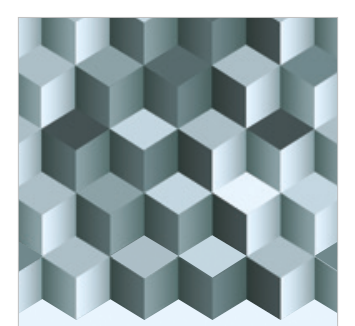

Journal of

Function Spaces

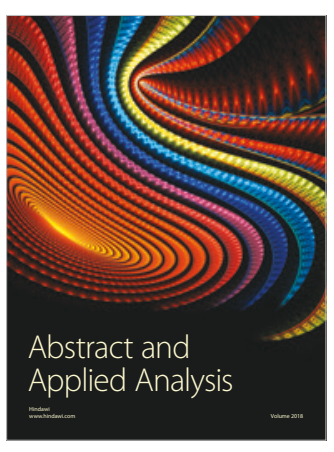

The Scientific

World Journal

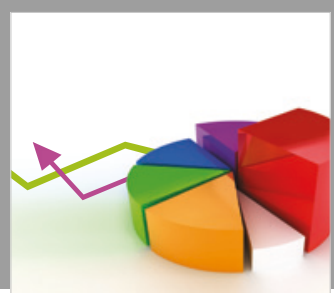

Journal of

Probability and Statistics
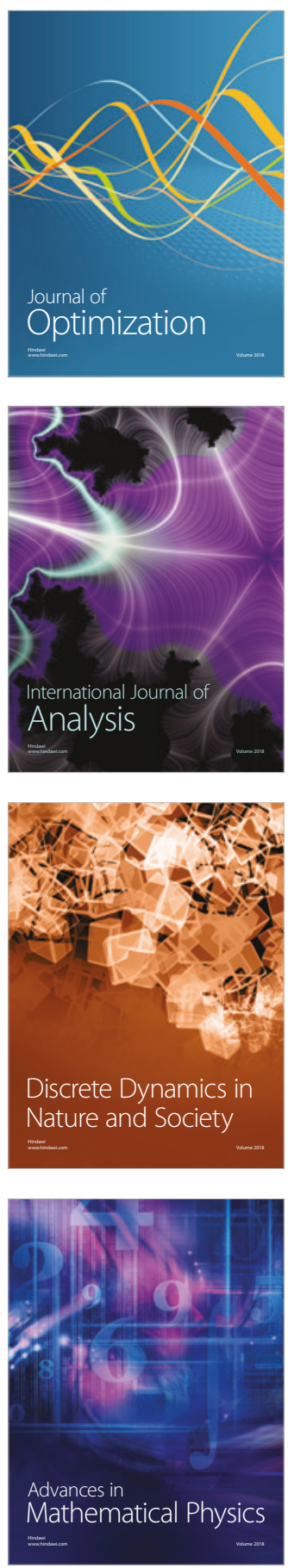